\title{
The provision of current and future Healthy Weight Management services from community pharmacies: A survey of community pharmacists' attitudes, practice and future possibilities
}

\author{
R. Newlands ${ }^{1}$, M. C. Watson ${ }^{2}$ and A. J. Lee ${ }^{2}$ \\ ${ }^{1}$ Section of Population Health, University of Aberdeen, Institute of Applied Health Sciences, University of Aberdeen, \\ Aberdeen AB25 2ZD, UK and ${ }^{2}$ Centre for Academic Primary Care, University of Aberdeen, Westburn Road, Aberdeen, \\ Scotland, AB25 2AY, UK
}

Obesity is acknowledged as a huge public health issue in Scotland with $66.4 \%$ of men and $59.6 \%$ of women are either overweight or obese $^{(1)}$. In Grampian, it is also one of the main public health concerns, where $62 \%$ of males and $55 \%$ of females are overweight or obese $^{(2)}$, and the cost of obesity ranks here second to smoking as a cause of its burden upon the health service ${ }^{(3)}$. The potential contribution of community pharmacy in public health, to prevent ill-health has been well recognised. Unlike England and Wales, there is no strategy for community pharmacies in Scotland to tackle obesity ${ }^{(3)}$. Therefore, the extent to which community pharmacies contribute to the management of obesity is unclear.

This study was a postal survey, sent out to all (128) community pharmacies in Grampian (May-June 2009) to determine which Healthy Weight Management (HWM) services were being provided, assess community pharmacists' activities and attitudes towards the provision of current and future HWM services, and also their training needs and barriers associated with the delivery of these services. The reliability of the survey was also determined by sending a shorter version of the original questionnaire within 2 weeks of their original reply.

This study achieved a good response rate $(83 / 128,64.8 \%)$. A number of services were already on offer, e.g. supply of weight loss medication $(n 69,84.1 \%)$ and advice about its use $(n 68,84.0 \%)$, dietary advice $(n 59,72.8 \%)$, physical activity advice $(n 53,66.3 \%)$ and BMI calculation ( $n 56,68.3 \%$ ). Pharmacists' attitude towards the provision of future HWM services was also found to be positive. They were mostly confident in measuring weight $(n 78,93.9 \%)$, height $(n 78,93.9 \%)$ and BMI $(n 78,93.9 \%)$. The identified barriers included: workload $(n 77,92.8 \%)$, the need for additional reimbursement $(n 63,75.9 \%)$ and additional staff $(n$ 49, 59.7\%). Few respondents had received training about HWM services either during undergraduate $(21.7 \%, n 18)$ or postgraduate $(26.5 \%, n 22)$ degrees. The most frequently identified training needs included estimation of body fat $(n 67,81.7 \%)$, one-to-one consultation skills $(n 60,73.2 \%)$ and advice on weight loss products ( $52,63.4 \%)$.

If barriers can be overcome and training needs addressed, community pharmacies and pharmacists could help to tackle the obesity problem in Grampian by providing more HWM services.

1. The Scottish Government (2010) Health of Scotland's population - Healthy Weight. http://www.scotland.gov.uk/Topics/statistics/browse/health/ trendobesity

2. ScotPHN Report. Scottish Action Obesity Resource. A project by scot PHN (Scottish Public Health Network) undertaken by Nutrition communication. Glasgow: ScotPHN; 2007.

3. Wilkie L. Annual Report of the Director of Public Health, Obesity, NHS Grampian, 2005/2006. http://www.nhsgrampian.org/nhsgrampian//files/ DPHannualreport.pdf 